\title{
Prevalence and predictors of overweight and obesity among women in the Gaza strip-Palestine: a cross-sectional study
}

\author{
Rima Rafiq El Kishawi ${ }^{1 *}$, Kah Leng Soo ${ }^{2}$, Yehia Awad Abed ${ }^{1}$ and Wan Abdul Manan Wan Muda ${ }^{2}$
}

\begin{abstract}
Background: The prevalence of overweight and obesity among women of childbearing age is considered a public health concern. Few studies have been conducted in the Gaza Strip to determine the magnitude of overweight and obesity. This study aimed to determine the prevalence of overweight and obesity along with their associated factors among women in the Gaza Strip.

Methods: A cross-sectional study was conducted to recruit a total of 357 mothers aged 18-50 years. Interviews were carried out among mothers to collect sociodemographic information, nutritional information, and physical activity. Anthropometric measurements [height, weight and waist circumference (WC)] were conducted with the mothers. Body Mass Index (BMI) was computed to determine the prevalence of overweight and obesity. Multinomial logistic regression was used to examine the associated factors of overweight and obesity.
\end{abstract}

Results: The combined prevalence of overweight and obesity among mothers was (64.1\%). The results of multinomial logistic regression showed the risk of overweight and obesity increased with age, the highest risk being in mothers aged $>33.0$ years $(\mathrm{OR}=2.7,95 \% \mathrm{Cl}:(1.06,6.86))$, and $(\mathrm{OR}=5.72,95 \% \mathrm{Cl}:(2.07,15.78))$, respectively, compared to mothers aged $<33.0$ years. Moreover, mothers with medium and high educational levels had a slightly higher risk of obesity $(\mathrm{OR}=0.31,95 \% \mathrm{Cl}:(0.15,0.64))$, and $(\mathrm{OR}=0.32,95 \% \mathrm{Cl}:(0.12,0.82))$ respectively than mothers with low educational level. Household income was positively associated with overweight and obesity. Mothers exposed to higher monthly income were more likely to be overweight or obese (OR=2.64, 95\% Cl: (1.20, $5.83))$, and $(\mathrm{OR}=3.06,95 \% \mathrm{Cl}:(1.28,7.29))$, respectively. Nutrition knowledge was significantly associated with a high prevalence of obesity $(\mathrm{OR}=1.20,95 \% \mathrm{Cl}:(1.03,1.38))$.

Conclusions: This study showed a higher prevalence of overweight and obesity among Palestinian women than previous studies. Age, educational level, monthly income, and nutrition knowledge were associated with the prevalence of overweight and obesity, compared to other variables that were not associated with overweight and obesity such as location, work status, physical activity, and sitting hours. Urgent action is needed to tackle overweight and obesity among women. Effective intervention is required to increase nutrition knowledge among women to improve their eating behaviors.

Keywords: Body mass index, Obesity, Overweight, Prevalence, Associated factors

\footnotetext{
* Correspondence: rimaa2_us@yahoo.com

${ }^{1}$ School of Public Health, Al Quds University, Gaza City, Gaza Strip, Palestine

Full list of author information is available at the end of the article
} 


\section{Background}

Obesity is commonly defined as a condition of abnormal or excessive fat storage in the adipose tissue to the extent of health impairment [1]. Excess weight is considered a serious public health concern contributing to several preventable chronic diseases such as diabetes, hypertension, heart disease and stroke, different types of cancers, osteoarthritis, and reproductive conditions [2]. Body Mass Index (BMI) is the most widely used tool to measure overweight and obesity [1]. On the other hand, waist circumference (WC) is used as a convenient indicator of abdominal adiposity, and it is a better predictor of the risk of chronic diseases than BMI [3].

The prevalence of overweight and obesity among women of childbearing age is considered a public health concern. Maternal obesity can result in negative outcomes for both women and fetuses. The maternal risks during pregnancy include gestational diabetes mellitus (GDM), preeclampsia and caesarean delivery. While, the fetus is at risk for stillbirth and congenital anomalies [4]. Globally, the prevalence of obesity has reached a point at which $50.0 \%$ of women of childbearing age are either overweight or obese [5]. According to World Health Organization (WHO) in 2016, 39.0\% of adults ( $\geq 18$ years) were overweight and $13.0 \%$ were obese [6]. Obesity prevalence is high in both the high-income and lowto-middle-income countries, but the prevalence of obesity is expected to increase more rapidly in the latter [7]. The main factor contributing to overweight and obesity is the imbalance between energy intake and energy expenditure [8, 9]. Worldwide, people consume more quantities of foods with a high energy density and exhibiting low physical activity, so this could lead to overweight and obesity epidemics around the world [10, 11]. Several variables associated with obesity, including genetic and demographic variables, such as a family history of obesity, age, and sex, cannot be modified; however, obesity-associated lifestyle factors (diet and physical activity) are often modifiable [12]. Patterns of food intake and eating habits in the nearby Eastern Mediterranean Region have changed noticeably during the past four decades [13]. Available studies on Eastern Mediterranean countries showed that obesity has reached an alarming level in both children and adults [14]. During the last three decades, the prevalence of overweight and obesity has increased at an alarming rate in the Arab countries, and it is more noticeable in women [15]. Palestinian adults are not spared from overweight and obesity and the recent trends have shown that people at the middleage are at the most risk of overweight and obesity especially among women [16]. The Palestinian community has been experiencing a nutrition transition, where studies have shown a high prevalence of nutrition-related chronic diseases such as heart diseases, diabetes, hypertension, and cancer, and their risk factors (lifestyle, and obesity) [17]. A study conducted in the Gaza Strip showed that the trends of overweight and obesity among Palestinian women in an urban area, a refugee camp, and a rural area were: $57.0,66.8$, and $67.5 \%$, respectively [18]. In the Gaza Strip, few studies have been conducted to determine the magnitude of overweight and obesity. Therefore, the objective of this study was to determine the prevalence of overweight and obesity among women in the Gaza Strip and to examine their association with the independent factors.

\section{Methods \\ Study design}

A cross-sectional study was performed to determine the prevalence of overweight and obesity among women in the Gaza Strip along with their associated factors. Data were collected between April and October 2012. Face to face interviews and anthropometric measurements were carried out to eligible women of the selected households.

\section{Sample size}

The sample size was calculated using the single proportion formula in the Epi-Info Software Revision (Version 2; 2002). Anticipated population prevalence of obesity $(\mathrm{p})=$ $32.0 \%$, which was among women in the Gaza Strip in 2008 [Al-Majdalawi J, Abed Y. Determinants of obesity among married women attended $\mathrm{MCH}$ clinics-Gaza Strip, unpublished Master thesis - Al Quds University], and level of significance $=5.0 \%(0.05)$. Therefore, the final number of $n=334$. To account for an attrition rate $20.0 \%$, the participants had to be recruited for this study as follows: $334+(0.20 \times 334)=400$ mothers.

A total of 400 households were selected and from each household a participant was recruited who met the inclusion and exclusion criteria of being a mother aged 18 to 50 years old and not pregnant.

Out of 400 households, 357 mothers were recruited with a response rate of $89.2 \%$. Among the participants, 12 mothers refused to participate in the study, and we excluded 31 households who did not meet the inclusion criteria as 18 mothers were pregnant, and 13 households did not include mothers' ages $18-50$ years.

\section{Sampling method}

A multi-stage cluster sampling process was used to find participants who met the inclusion and exclusion criteria of a mother aged 18 to 50 years who was not pregnant or lactating to avoid subject bias. The Gaza Strip is administratively divided into main five districts, of which three districts were selected: north of Gaza Strip, Gaza city, and south of the Gaza Strip [19].

Three clusters were chosen randomly from those three districts depending on the sociodemographic situation: 
Jabalia as refugee camp in the north of the Gaza Strip, and El Remal as an urban area in Gaza city, then Al Qarara as a rural area in the South of Gaza Strip. At the first stage, a number of areas were chosen randomly from the entire of each cluster, then, systematic random sample households were selected within each area in the urban, the refugee camp, and the rural respectively. The number of households selected from each cluster was weighted in proportion to the total population of women in childbearing age (19.1\%). Hence, the distribution of households in each area was 220 from Jabalia camp, 140 from El Remal area, and 40 from Al Qarara (Table 1).

\section{Data collection}

Data were collected using a structured questionnaire for face to face interviews. Validity of the questionnaire was checked and obtained by consulting a number of experts with experience and knowledge of the topic to make suggestions and judgment about the adequacy of the tools. Interviews were carried out among mothers to collect information on a number of predictors which could potentially affect the nutritional status of women such as sociodemographic information, nutrition information, and physical activity. The variables which are included in this study are: Socioeconomic and demographic data as follow: Area of residence was divided into urban, rural and refugee camp. Household monthly income and it was expressed in New Israel Shekel (NIS) as NIS 3.9 was equal to US\$1. Household income was categorized into three classes $(<1000$ NIS, 1000-2000 NIS and $>2000$ NIS). Food or financial assistance was received from non-government agencies like UNRWA (United Nations Relief and Works Agency). Mothers were asked their age at their most recent birthday and it was categorized as $(\leq 26,27-33,>33)$ years. Education level was defined as low level (elementary or below and preparatory), medium level (secondary); and high level (diploma/university level, and postgraduate). The mothers were asked about family size (number of family members), and the number of pregnancies.

The International Physical Activity Questionnaire (IPAQ) has been utilized internationally to obtain comparable estimates of physical activity patterns [20]. To measure the physical activity pattern of mothers, the researchers used the validated Arabic short-format version of the IPAQ. The questions were about the time mothers spent being physically active in the last 7 days, including questions about activities mothers did at work, as part of household chores to get from place to place, and in their spare time for recreation, exercise, or sport. In answering the questions, vigorous physical activities referred to activities that required vigorous physical effort and made mothers breathe much harder than normal. In contrast, moderate physical activities referred to activities that required moderate physical effort and made mothers breathe relatively harder than normal. Data collected with IPAQ can be reported as a continuous score. An average metabolic equivalent of task (MET) score was derived for each type of activity. In this study, sitting time is used as an independent proxy measure of sedentary behavior [21]. This measure included time spent sitting (in days and minutes/day) at work, at home, while doing coursework, and during leisure time (sitting at a desk, visiting a friend, reading, and watching TV). The nutrition knowledge of a participant was assessed using an adapted questionnaire from the National Coordinating Committee for Food and Nutrition, Ministry of Health, Malaysia, 1997. The questionnaire consisted of 16 questions included 5 categories addressing: food nutrients sources, different types of healthy food, healthy food and body functions, body weight and exercise, and food and its related to diseases. The median of the nutritional knowledge scores $=13$, it was used to separate the higher half of mothers' scores distribution $\geq 13$, from the lower half $<13$. Validity of the questionnaire was checked and obtained by consulting a number of experts in health and nutrition from Al-Quds University, and the United Nations Children's Fund (UNICEF) in the Gaza Strip. In addition, Kuder-Richardson Formula 20 (KR-20) was calculated for each item and the general reliability for all items was 0.76 . It was considered acceptable, as the acceptable value was from 0.70 to 0.95 .

Pilot study questionnaires were distributed among 30 women before conducting the study, and after obtaining the results, the necessary changes were made. Anthropometric measurements were taken by two trained research assistants. The heights and weights of mothers were measured using the standard recommended procedures of the WHO [22]. SECA digital weighing scale (to the nearest $0.1 \mathrm{~kg}$ ) and SECA body meter (with a precision of $0.1 \mathrm{~cm}$ ) were used to measure the heights and

Table 1 Sample distribution

\begin{tabular}{llll}
\hline Area & Estimated population & Estimated mothers & Sample \\
\hline Jabalia (Refugee Camp) & 110,000 & 21,010 & 220 \\
El Remal (Urban area) & 70,000 & 13,370 & 140 \\
Al Qarara (Rural area) & 20,000 & 3820 & 40 \\
Total & 200,000 & 38,200 & 400 \\
\hline
\end{tabular}


the weights of the mothers, respectively, and mothers wore lightweight clothes and no shoes. The Body Mass Index (BMI) was computed with the following formula: weight $(\mathrm{kg}) /$ height $(\mathrm{m})^{2}$. According to $\mathrm{WHO}$, an adult BMI of less than 18.5 was considered to be underweight, between 18.5 and 24.9 was normal, and between 25.0 and 29.9 was overweight. An adult with a BMI of 30.0 or higher was considered obese [1]. The waist measurement was obtained with a tape measure to the nearest $0.1 \mathrm{~cm}$, with mothers wearing only light clothing around the waist area in a horizontal plane, midway between the inferior margin of the ribs and the superior border of the iliac crest. The WHO recommends sexspecific WC cut-off points for women $(\geq 80 \mathrm{~cm})$, thus, women with WCs of $>88 \mathrm{~cm}$, face a high risk of cardiovascular disease (CVD) [23].

\section{Data analysis}

Analyses were performed with the statistical software package version (SPSS) 22.0. Descriptive statistics such as frequency and proportion were used to describe the sample and to determine the prevalence of overweight and obesity among women in the Gaza Strip. Cross tabulations and Pearson's Chi-square test were used to obtain the associations and strength of relationship between the independent and the dependent variables. Multinomial logistic regression analysis was performed to control for confounding factors and to determine the Odds Ratios (ORs). The univariate analysis was carried out to evaluate each independent variable for its unadjusted association with overweight or obesity. In the second, bivariate associations, we included all independent variables with $p<0.25$ significantly associated with overweight or obesity, because they were considered important to evaluate factors associated with overweight or obesity. In the final model, the significance level was $p<0.05$.

\section{Ethical issues}

Ethical approval was obtained from the Helsinki Committee for Research Ethics in the Ministry of Health in the Gaza Strip, and ethical clearance was approved from University Sains Malaysia (USM) ethical committee. The written consent form was obtained from each mother before the interview. Moreover, mothers were informed that the questionnaires could be answered voluntarily, and that the information would be treated confidentially.

\section{Results}

Out of 400 households, 357 were identified and screened to find mothers who met the inclusion and exclusion criteria and agreed to participate in this study giving a response rate of $89.2 \%$.
Table 2 Mothers' Anthropometric characteristics $(n=357)$

\begin{tabular}{lllc}
\hline Variables & Frequency(n) & Percent (\%) & Mean (SD) \\
\hline Mother's body weight $(\mathrm{kg})$ & & & $70.95(15.46)$ \\
Mother's body height $(\mathrm{m})$ & & & $1.59(0.05)$ \\
Waist circumference $(\mathrm{cm})^{\mathrm{a}}$ & & & $91.12(12.76)$ \\
$\quad<80.0$ & 62 & 17.4 & \\
$\geq 80.0$ & 295 & 82.6 & $27.9(5.91)$ \\
Mother's BMI & & & \\
Underweight <18.5 & 7 & 2.0 & \\
Normal weight 18.0-24.9 & 121 & 33.9 & \\
Overweight 25.0-29.9 & 123 & 34.5 & \\
Obese class I 30.0-34.9 & 60 & 16.2 & \\
Obese class II 35.0-39.9 & 34 & 9.5 & \\
Obese class III $\geq 40.0$ & 12 & 3.9 & \\
aRef. [23] & &
\end{tabular}

Table 2 presents the mothers' anthropometric characteristics. The mean \pm SD weight and height of the mothers were $70.95 \pm 15.46 \mathrm{~kg}$ and $1.59 \pm 0.05 \mathrm{~m}$, respectively. The mean \pm SD WC was $91.12 \pm 12.76 \mathrm{~cm}$ and the mean \pm SD BMI was $27.90 \pm 5.91 \mathrm{~kg} / \mathrm{m}^{2}$. The results revealed that the combined prevalence of overweight and obesity was $64.1 \%$. The prevalence of overweight/obesity was 34.5 and $29.6 \%$ respectively. Table 3 shows the prevalence of overweight and obesity increased as age increased, with the highest prevalence 33.3, and $47.7 \%$ respectively in the age $>33$-years, $(p<0.001)$. The results showed that weight increased as income increased, the prevalence of overweight and obesity was significantly higher in participants with high family income 38.0 and $33.8 \%$ respectively, compared to those with low family income, $(p=0.022)$. Moreover, the prevalence of overweight and obesity was high 35.3 and $41.9 \%$ among mothers with high number of pregnancies, $(p=0.001)$. A multinomial logistic regression model was used to assess the odds for the sociodemographic and other variables to a participant being overweight and obese (Table 4). Our results revealed that as the age of mothers increased especially $>33$ years, the risk of being overweight and obese increased ( $\mathrm{OR}=$ 2.70; 95\% CI: $1.06-6.86 ; p=0.038)$ and $(\mathrm{OR}=5.72,95 \%$ : $2.07,15.78 ; p=0.001$ ), respectively. In addition, higher educated mothers had a slightly higher risk of obesity than mothers with lower educational levels $(\mathrm{OR}=0.32$; 95\% CI: $0.12-0.82 ; p=0.017)$. The results revealed that mothers lived in households with higher monthly income (> 2000 NIS) or (1000-2000NIS) appeared to have significantly higher risks of being overweight $(\mathrm{OR}=$ $2.64 ; 95 \% \mathrm{CI}: 1.20-5.83 ; p=0.015)$ or $(\mathrm{OR}=2.58 ; 95 \%$ CI: $1.40-4.74 ; p=0.002)$ than those with lower monthly income $(<1000 \mathrm{NIS})$. Moreover, our findings further revealed that mothers lived in households with higher 
Table 3 Baseline characteristics of mothers in the Gaza Strip by BMI category $(n=357)$

\begin{tabular}{|c|c|c|c|c|}
\hline Variables & $\begin{array}{l}\text { Normal weight } \\
(\boldsymbol{n}=128)\end{array}$ & $\begin{array}{l}\text { Overweight } \\
(\boldsymbol{n}=123)\end{array}$ & $\begin{array}{l}\text { Obesity } \\
(\boldsymbol{n}=106)\end{array}$ & $\boldsymbol{P}$-Value $\left(X^{2}\right)$ \\
\hline Geographic location & & & & 0.107 \\
\hline Urban & $43(43.0)$ & $26(26.0)$ & $31(31.0)$ & \\
\hline Rural & $13(32.5)$ & $19(47.5)$ & $8(20.0)$ & \\
\hline Refugee camp & $72(33.2)$ & $78(35.9)$ & $67(30.9)$ & \\
\hline Age (year) & & & & $<0.001(42.73)^{*}$ \\
\hline$\leq 26$ & $57(55.9)$ & $31(30.4)$ & $14(13.7)$ & \\
\hline $27-33$ & $50(34.7)$ & $55(38.2)$ & $39(27.1)$ & \\
\hline$>33$ & $21(19.0)$ & $37(33.3)$ & $53(47.7)$ & \\
\hline Educational level & & & & $0.017(12.01)^{*}$ \\
\hline High & $29(36.7)$ & $32(40.5)$ & $18(22.8)$ & \\
\hline Medium & $60(42.9)$ & $46(32.8)$ & $34(24.3)$ & \\
\hline Low & $39(28.3)$ & $45(32.6)$ & $54(39.1)$ & \\
\hline Work status & & & & 0.294 \\
\hline Housewife & $122(36.0)$ & $114(33.6)$ & $103(30.4)$ & \\
\hline Employee & $6(33.3)$ & $9(50.0)$ & $3(16.7)$ & \\
\hline Household member & & & & 0.090 \\
\hline $3-6$ & $92(49.5)$ & $61(32.8)$ & $33(17.7)$ & \\
\hline$>6$ & $36(21.1)$ & $62(36.3)$ & $73(42.6)$ & \\
\hline Household assistance & & & & 0.492 \\
\hline Yes & $63(34.1)$ & $62(33.5)$ & $60(32.4)$ & \\
\hline No & $65(37.8)$ & $61(35.5)$ & $46(26.7)$ & \\
\hline Monthly income (NIS) & & & & $0.022(11.49)^{*}$ \\
\hline$>2000$ & $20(28.2)$ & $27(38.0)$ & $24(33.8)$ & \\
\hline $1000-2000$ & $43(29.7)$ & $58(40.0)$ & $44(30.3)$ & \\
\hline$<1000$ & $65(46.0)$ & $38(27.0)$ & $38(27.0)$ & \\
\hline Nutrition knowledge score & & & & 0.078 \\
\hline$<13$ & $63(38.4)$ & $62(37.8)$ & $39(23.8)$ & \\
\hline$\geq 13$ & $65(33.7)$ & 61 (31.6) & $67(34.7)$ & \\
\hline Type of physical activity & & & & 0.203 \\
\hline Low & $23(29.9)$ & $25(32.5)$ & 29 (37.6) & \\
\hline Moderate & $105(37.5)$ & $98(35.0)$ & $77(27.5)$ & \\
\hline \multicolumn{5}{|l|}{ Sitting time (hour/day) } \\
\hline $1-3$ & $94(37.5)$ & $84(33.4)$ & $73(29.1)$ & 0.623 \\
\hline$>3$ & $34(32.1)$ & $39(36.8)$ & $33(31.1)$ & \\
\hline Number of pregnancies & & & & $<0.001(30.88)^{*}$ \\
\hline $1-5$ & $90(47.4)$ & $64(33.7)$ & $36(18.9)$ & \\
\hline$>5$ & $38(22.8)$ & $59(35.3)$ & $70(41.9)$ & \\
\hline
\end{tabular}

Chi square test. *Statistically significant $P$-value $<0.05$

$1 \$=3.9 \mathrm{NIS}$

High Educational level: Diploma, university level, and master's degree/PhD; Medium Educational level: secondary; Low Educational level: elementary or below and preparatory

household income (>2000NIS) or (1000-2000NIS) were significantly associated with higher rates of obesity $(\mathrm{OR}=3.06$; 95\% CI: $1.28-7.29 ; p=0.011)$, or $(\mathrm{OR}=2.36$;
95\% CIs: $1.21-4.62 ; p=0.012)$, respectively than those with lower monthly income $(<1000 \mathrm{NIS})$. In addition, our data (Table 4) showed that as the scores of 
Table 4 Factors associated with body mass index (overweight and obesity) based on a multinomial analysis

\begin{tabular}{|c|c|c|c|c|c|c|}
\hline \multirow[t]{2}{*}{ Variables } & \multicolumn{2}{|c|}{ Overweight ( $\boldsymbol{n}=123$ ) } & \multicolumn{4}{|c|}{ Obesity $(\boldsymbol{n}=106)$} \\
\hline & $\mathrm{OR}$ & $(95 \% \mathrm{Cl})$ & $\boldsymbol{P}$-value & OR & $(95.0 \% \mathrm{Cl})$ & $\boldsymbol{P}$-value \\
\hline \multicolumn{7}{|l|}{ Age (year) } \\
\hline$\leq 26$ & 1 & - & - & 1 & - & - \\
\hline $27-33$ & 1.80 & $(0.91,3.56)$ & 0.092 & 2.21 & $(0.97,5.02)$ & 0.057 \\
\hline$>33$ & 2.70 & $(1.06,6.86)$ & $0.038^{*}$ & 5.72 & $(2.07,15.78)$ & $0.001^{*}$ \\
\hline \multicolumn{7}{|l|}{ Educational level } \\
\hline Low & 1 & - & - & 1 & - & - \\
\hline Medium & 0.56 & $(0.29,1.08)$ & 0.086 & 0.31 & $(0.15,0.64)$ & $0.001^{*}$ \\
\hline High & 0.80 & $(0.35,1.83)$ & 0.600 & 0.32 & $(0.12,0.82)$ & $0.017^{*}$ \\
\hline \multicolumn{7}{|c|}{ Monthly income NIS (1\$= 3.9NIS) } \\
\hline$<1000$ & 1 & - & - & 1 & - & - \\
\hline $1000-2000$ & 2.58 & $(1.40,4.74)$ & $0.002^{*}$ & 2.36 & $(1.21,4.60)$ & $0.012^{*}$ \\
\hline$>2000$ & 2.64 & $(1.20,5.83)$ & $0.015^{*}$ & 3.06 & $(1.28,7.29)$ & $0.011^{*}$ \\
\hline Nutrition knowledge & 1.10 & $(0.95,1.22)$ & 0.243 & 1.20 & $(1.03,1.38)$ & $0.016^{*}$ \\
\hline
\end{tabular}

nutrition knowledge increased to a participant, the risk of being obese increased $(\mathrm{OR}=1.20 ; 95 \% \mathrm{CI}$ : $1.03-$ $1.38 .0 ; p=0.016)$.

\section{Discussion}

The combined prevalence of overweight and obesity among women aged 18 to 50 years is high (64.1\%). While a previous study reported that $71.3 \%$ of Palestinian women aged 30 to 65 years were overweight and obese [24]. The differences in results can be attributed to variation in age groups. In the developing countries, obesity is not only a condition of high socioeconomic status (SES) groups, but the risks of obesity tend to shift to the lower SES groups towards the poorer groups [25, 26]. Our results revealed that the prevalence of obesity is $29.6 \%$, which is a high trend associates with a high risk of non-communicable diseases. Palestinian women were observed to be particularly vulnerable to obesity-related diseases along with co-morbidities like diabetes [17]. In addition, the common abnormality in our participants was the majority of women had abnormal WC, which is a convenient indicator of abdominal obesity and it is related to cardiovascular risk factors [2]. Based on the multinomial logistic regression analysis, overweight and obesity increased as age increased. Our results revealed that the highest prevalence of overweight and obesity was seen among women in the age group ( $>33$ years). These findings are consistent with other studies reported that the prevalence of obesity increased with age [27, 28 ]. The association between excess weight and age can be explained by the physiological and behavioral changes within women that occur as a result of aging like the lowering in energy requirement at rest with aging [29, 30]. This generally indicates that women at an older age with decreasing physical activity help accumulate more weight [28]. Education was a typical indicator of SES associated with excess weight in developed countries, while income was in the developing countries [31]. The results of this study revealed that women with higher educational level had a slightly higher risk of being obese than women with lower educational level. Similar results reported that individuals with higher education levels were more likely to be overweight $[28,32]$. Higher education helps women live a life that includes less physical activity and helps access energy-dense food which is considered to cause overweight or obesity [28]. While in other studies, results revealed that high prevalence of obesity was found among people who were less educated. As it is known that people with higher levels of education are healthier than people with lower levels of education [33]. Education is associated with awareness and attitude attainment that allows people to integrate healthy behaviors into their lifestyle [26]. Interestingly, in this study, nutrition knowledge was not a protective factor for obesity, it was found in the multinomial analysis that those with high nutrition knowledge scores were at high risk to be obese than those with low nutrition knowledge scores. That could be due to such knowledge wasn't translated into good practices. Nutritional knowledge is positive when it's translated to practice and results in better nutrition; otherwise, it is considered deceased/less knowledge [34]. A previous study was 
conducted in the United Kingdom showed that, despite a high level of awareness on healthy eating and many public health campaigns to encourage people to adopt healthy diets, obesity prevalence still increased [35]. Our results further revealed that the household's income was positively associated with overweight and obesity. This study showed that women from non-poor households have significantly increased the risk of overweight and obesity than those belonging to poor families. It was suggested that high economic status was significantly associated with weight gain. The same results of other studies showed that a significant positive relationship between income and a high BMI $[28,36]$. That could be explained by high calories food intake, with a lack of awareness of the health risk associated obesity, as well as a more sedentary lifestyle which is considered to cause weight gain [37].

However, our results revealed that all women were low or moderately active, but there was no significant association between physical activity and excess weight. [Abdeen et al. (2012)] recognized in his study low physical activity among Palestinian obese women, and that could be due to the limited availability of exercising facilities for Palestinian girls and women [16]. A similar result has been found in Arab countries, specifically, the prevalence of a sedentary lifestyle increased along with the nutritional transition, especially with the advancements in technology and transportation [13]. Women in most Arab states generally faced more barriers to the practice of physical activity than men because of social traditions and safety reasons. In Bahrain, $67.0 \%$ of women perceived that they had limited opportunities to participate in physical activity because most exercises and sports facilities are only provided for men [38]. This study has some limitations which have to be pointed out. The study design was cross-sectional to identify associated factors with overweight and obesity, which could not determine the cause and effect relationship. Case-control studies should be conducted in the future to address the risk factors of weight gain. In this study, we assessed physical activity as a subjective tool (IPAQ), instead of a more objective tool such as an accelerometer or pedometer.

\section{Conclusions}

Our findings revealed a higher prevalence of overweight and obesity among Palestinian women than previously reported in other local studies. Therefore, obesity is a major public health problem among women in the Gaza Strip. Age, educational level, monthly income, and nutritional knowledge were associated with overweight and obesity among women in the Gaza Strip. There is a need for urgent action to develop health strategies to combat the growth of overweight and obesity. In addition, an effective nutrition education program should be implemented to help women to convey their nutrition knowledge into healthy dietary practices.

\section{Abbreviations}

BMI: Body mass index; Cl: Confidence interval; CVD: Cardiovascular disease; IPAQ: International physical activity questionnaire; OR: Odds ratio; MET: Metabolic equivalent of task; SES: Socioeconomic status; SPSS: Statistical package for social science; SD: Standard deviation; UNCIF: United Nations children's fund; WC: Waist circumference; WHO: World health organization

\section{Acknowledgements}

We thank all participants from the three areas in the Gaza Strip for their free participation in this study.

\section{Authors' contributions}

R.R El Kishawi designed the study, entered and analyzed the data. WAM. Wan Muda participated in the design of this study and the main supervisor of this research. KL. Soo participated in data analysis. Y. A. Abed provided content advice. All authors participated in the review of the manuscripts and approved the final version.

\section{Funding}

No funding for this study.

\section{Availability of data and materials}

Data are available from the authors upon reasonable request.

\section{Ethics approval and consent to participate}

Ethical approval was obtained from the Helsinki Committee for Research Ethics in the Ministry of Health in the Gaza Strip, and ethical clearance was approved from University Sains Malaysia (USM) ethical committee. The written consent form was obtained from each mother before the interview. Moreover, mothers were informed that the questionnaires could be answered voluntarily, and that the information would be treated confidentially.

\section{Consent for publication}

Not applicable.

\section{Competing interests}

The authors declare that they have no significant competing professional, financial, or personal interests that might have influenced the performance of the work described in this manuscript.

\section{Author details}

${ }^{1}$ School of Public Health, Al Quds University, Gaza City, Gaza Strip, Palestine. ${ }^{2}$ Program of Nutrition, School of Health Sciences, Health Campus, Universiti Sains Malaysia, 16150 Kubang Kerian, Kelantan, Malaysia.

Received: 10 January 2020 Accepted: 20 May 2020

Published online: 05 June 2020

\section{References}

1. WHO. "Obesity: preventing and managing the global epidemic", Technical Report Series, No. 894. Geneva: World Health Organization; 2000.

2. WHO. Comparative Quantification of Health Risks: Global and Regional Burden of Disease Attributable to Selected Major Risk Factors. Geneva: World Health Organization; 2004.

3. Schunkert H, Markus MRP, Stritzke J. Waist circumference and cardiovascular risk. Handbook of Anthropometry: Springer; 2012. p. 2137-53.

4. Hashmi A, Soomro JA, labal Z, Soomro TK, Saleem K. Maternal Obesity: A Global Health Problem and It's Implications on Maternal and Fetal Health. J Family Reprod Health. 2010;(4):155-60.

5. Tenenbaum-Gavish K, Hod M. Impact of maternal obesity on fetal health. Fetal Diagn Ther. 2013;34(1):1-7.

6. WHO, "Obesity and overweight", World Health Organization, Geneva, 3 March 2020. https://www.who.int/news-room/fact-sheets/detail/obesity-andoverweight. Accessed 15 Mar.2020. 
7. Ford ND, Patel SA, Narayan KV. Obesity in low-and middle-income countries: burden, drivers, and emerging challenges. Annu Rev Public Health. 2017;38:145-64.

8. Fonseca DC, Sala P, BdAM F, Reis J, Torrinhas RS, Bendavid I, Dan Linetzky Waitzberg DL. Body weight control and energy expenditure. Clin Nutr Exp. 2018;20:55-9.

9. Romieu I, Dossus L, Barquera S, Blottière HM, Franks PW, Gunter M, et al. Energy balance and obesity: what are the main drivers? Cancer Causes Control. 2017;28(3):247-58

10. Fisberg M, Maximino P, Kain J, Kovalskys I. Obesogenic environmentintervention opportunities. J Pediatr. 2016;92(3):30-9.

11. WHO. Controlling the global obesity epidemic. 2017. World Health Organization. 2011. http://www.who.int/nutrition/topics/obesity/en/index. html. Accessed 24 Aug 2018.

12. Al-Hazzaa HM, Abahussain NA, Al-Sobayel HI, Qahwaji DM, Musaiger AO. Lifestyle factors associated with overweight and obesity among Saudi adolescents. BMC Public Health. 2012;12(1):354

13. Musaiger AO. Diet and prevention of coronary heart disease in the Arab Middle East countries. Med Princ Pract. 2002;11(Suppl 2):9-16.

14. Nasreddine L, Mokdad A, Adra N, Tabet M, Hwalla N. Nutrition transition and cardiovascular disease risk factors in Middle East and North Africa countries: reviewing the evidence. Ann Nutr Metab. 2010;57(3-4):193-203.

15. ALNohair S. Obesity in gulf countries. Int J Health Sci. 2014;8(1):79-83.

16. Abdeen Z, Jildeh C, Dkeideek S, Qasrawi R, Ghannam I, Al SH. Overweight and obesity among Palestinian adults: analyses of the anthropometric data from the first National Health and nutrition survey (1999-2000). J Obes. 2012;2012.

17. Mikki N, Abdul-Rahim H, Awartani F, Holmboe-Ottesen G. Prevalence and sociodemographic correlates of stunting, underweight, and overweight among Palestinian school adolescents (13-15 years) in two major governorates in the West Bank. BMC Public Health. 2009;9(1):485.

18. El Kishawi RR, Soo KL, Abed YA, Muda WAMW. Obesity and overweight: prevalence and associated socio demographic factors among mothers in three different areas in the Gaza strip-Palestine: a cross-sectional study. BMC Obes. 2014;1(1):7.

19. Palestinian Central Bureau Statistics. (2009). Population, Housing and Establishment Census-2007.Palestinian central bureau statistics.

20. The International Physical Activity. Short last 7 days self administrated format 2002. Retrieved October14, 2018, from http://www.ipaq.ki.se/

21. Sjöström M, Oja P, Hagströmer M, Smith B, Bauman A. Health-enhancing physical activity across European Union countries: the Eurobarometer study. J Public Health. 2006;14(5):291-300

22. WHO. Physical status: the use and interpretation of anthropometry. In: Report of a WHO Expert Committee. Technical Report Series No. 854. Geneva: World Health Organization; 1995.

23. WHO. Waist Circumference and Waist-Hip Ratio. Report of a WHO Expert Consultation. Geneva: World Health Organization; 2011.

24. Husseini A, Abu-Rmeileh NME, Mikki N, Ramahi TM, Ghosh HA, Barghuthi N, et al. Cardiovascular diseases, diabetes mellitus, and cancer in the occupied Palestinian territory. Lancet. 2009:373(9668):1041-9.

25. Hossain P, Kawar B, El Nahas M. Obesity and diabetes in the developing world-a growing challenge. N Engl J Med. 2007;356(3):213-5.

26. Monteiro CA, Moura EC, Conde WL, Popkin BM. Socioeconomic status and obesity in adult populations of developing countries: a review. Bull World Health Organ. 2004;82(12):940-6.

27. Chowdhury MAB, et al. Trends, prevalence and risk factors of overweight and obesity among women of reproductive age in Bangladesh: a pooled analysis of five national cross-sectional surveys. BMJ Open. 2018;8(7): e018468.

28. Gouda J, Prusty RK. Overweight and obesity among women by economic stratum in urban India. J Health Popul Nutr. 2014;32(1):79-88.

29. Erem C, Arslan C, Hacihasanoglu A, Deger O, Topbaş M, Ukinc K, Ersöz HO, Telatar M. Prevalence of obesity and associated risk factors in a Turkish population (Trabzon city, Turkey). Obes Res. 2004;12(7):1117-27.

30. Nooyens AC, Visscher TL, Verschuren WM, Schuit AJ, Boshuizen HC, van Mechelen W, Seidell JC. Age, period and cohort effects on body weight and body mass index in adults: the Doetinchem cohort study. Public Health Nutr. 2009;12(06):862-70

31. Sen J, Mondal N, Dutta S. Factors affecting overweight and obesity among urban adults: a cross-sectional study. Epidemiol Biostatistics Public Health. 2013;10(1).
32. Hu G, Pekkarinen $H$, Hänninen $\mathrm{O}$, Tian $H$, Jin R. Comparison of dietary and non-dietary risk factors in overweight and normal-weight Chinese adults. $\mathrm{Br}$ J Nutr. 2002;88(1):91-7

33. Fletcher JM, Frisvold DE. Higher education and health investments: does more schooling affect preventive health care use? J Hum Cap. 2009;3(2): 144-76.

34. Gittelsohn J, Vastine AE. Sociocultural and household factors impacting on the selection, allocation and consumption of animal source foods: current knowledge and application. J Nutr. 2003;133(11):4036S-41S.

35. O'Brien G, Davies M. Nutrition knowledge and body mass index. Health Educ Res. 2007;22(4):571-5.

36. Paeratakul S, Popkin BM, Keyou G, Adair LS, Stevens J. Changes in diet and physical activity affect the body mass index of Chinese adults. Int J Obes Relat Metab Disord. 1998;22:424-31.

37. Shayo GA, Mugusi FM. Prevalence of obesity and associated risk factors among adults in Kinondoni municipal district, Dar Es Salaam Tanzania. BMC Public Health. 2011;11(1):365.

38. Musaiger AO, Al-Mannai M, Tayyem R, Al-Lalla O, Ali EY, Kalam F, et al. Perceived barriers to healthy eating and physical activity among adolescents in seven Arab countries: a cross-cultural study. Sci World J. 2013;2013.

\section{Publisher's Note}

Springer Nature remains neutral with regard to jurisdictional claims in published maps and institutional affiliations.
Ready to submit your research? Choose BMC and benefit from:

- fast, convenient online submission

- thorough peer review by experienced researchers in your field

- rapid publication on acceptance

- support for research data, including large and complex data types

- gold Open Access which fosters wider collaboration and increased citations

- maximum visibility for your research: over $100 \mathrm{M}$ website views per year

At $\mathrm{BMC}$, research is always in progress.

Learn more biomedcentral.com/submissions 